\title{
Experimental Realization of Entanglement Concentration and A Quantum Repeater
}

\author{
Zhi Zhao ${ }^{1}$, Tao Yang ${ }^{1}$, Yu-Ao Chen ${ }^{1}$, An-Ning Zhang ${ }^{1}$ and Jian-Wei Pan ${ }^{1,2 *}$ \\ ${ }^{1}$ Department of Modern Physics, University of Science \\ and Technology of China, Hefei, Anhui 230027, China \\ ${ }^{2}$ Institut für Experimentalphysik, Universitat Wien, \\ Boltzmanngasse 5, 1090 Wien, Austria
}

(Dated: December 21, 2002)

\begin{abstract}
We report an experimental realization of entanglement concentration using two polarizationentangled photon pairs produced by pulsed parametric down-conversion. In the meantime, our setup also provides a proof-in-principle demonstration of a quantum repeater. The quality of our procedure is verified by observing a violation of Bell's inequality by more than 5 standard deviations. The high experimental accuracy achieved in the experiment implies that the requirement of tolerable error rate in multi-stage realization of quantum repeaters can be fulfilled, hence providing a practical toolbox for quantum communication over large distances.
\end{abstract}


In recent years, significant experimental advances have been achieved in the field of quantum communication (QC). In particular, entangled photon pairs have been used to experimentally demonstrate a number of QC schemes such as dense coding [1, 2], quantum teleportation [3, 4, 5, 6] and quantum cryptography [7, 8, 9, 10, 11]. Though the above schemes are achievable for moderate distances (up to a few tens of kilometers in quantum cryptography), serious problems occur in bringing QC to technologically useful scales. One of the problems is the photon losses in the transmission channel. In quantum cryptography, the photon losses by themselves only reduce the bit rate (exponentially with distance). With perfect detectors the distance would not be limited, one can solve this problem by sending more photons. However, due to the dark counts, whenever a photon is lost there is a chance that a dark count produces an error. Hence, when the probability of a dark count becomes comparable to the probability that a photon is correctly detected, the signal-tonoise ratio tends to 0. Fortunately, as shown in ref. [12] the use of entangled photons and of entanglement swapping [13] could offer ways to extend the achievable distances.

Another problem is caused by the noisy quantum channel. The decoherence degrades the quality of entanglement between two particles more and more the further they propagate. However, all the above protocols require that two distant parties, usually called Alice and Bob, share highly entangled photon pairs. The decoherence can be overcome by exploiting entanglement purification [14, 15] - a way to extract a subset of highly entangled states from a large set of less entangled states using local operations and classical communication. Therefore, quantum repeaters [16], a combination of entanglement swapping and entanglement purification, hold the promise to solve the problems of the photon losses and of the decoherence in long-distance QC.

Though entanglement swapping has been experimentally demonstrated with high accuracy [17, 18, 19], the implementation of quantum repeaters remains to be an experimental challenge due to the difficulty to achieve entanglement concentration. Various purification schemes have been proposed for pure or mixed entangled states. They mainly include three classes. First, the so-called Procrustean method [20]. It requires that the photon pairs are all in a pure non-maximally entangled state, say, $\alpha|H\rangle|V\rangle+\beta|V\rangle|H\rangle$. Here $\alpha$ and $\beta$ are two known coefficients that satisfy $|\alpha|^{2}+|\beta|^{2}=1$, and $|H\rangle$ and $|V\rangle$ denote horizontal and vertical polarizations of the photons. In this case, the scheme only involves local filtering operations on single pairs. Second, the so-called Schmidt decomposition scheme [20], in which the pho- 
ton pairs are all in a pure but unknown non-maximally entangled state $\alpha|H\rangle|V\rangle+\beta|V\rangle|H\rangle$. In practice, this scheme is difficult to implement, since it requires simultaneous collective measurements on many photons. To distinguish the second case from the others, in what follows we refer to it as entanglement concentration. Third, the general purification method that works for arbitrary mixed states 14, 15]. The implementation of the general scheme is more difficult as it requires controlled-NOT (CNOT) operations between different photons.

We emphasize that, though only the general scheme can be used to purify arbitrary mixed states, both local filtering and entanglement concentration are of interest in their own rights. This is because, on the one hand, both methods provide a way to generate maximally entangled states, which is different from the general scheme where only highly entangled states are generated. On the other hand, with the help of local filtering any inseparable states can be purified [21], while the general scheme alone only works for the cases where the entanglement fidelity $F$ is larger than $1 / 2[14]$.

Up to now, except for the Procrustean method has been demonstrated very recently [22], there is no experimental implementation of the other schemes due to the difficulty to control many photons. To avoid the difficulties caused by collective measurements and CNOT operations, several practical schemes have been proposed for entanglement concentration 23, 24] and entanglement purification [25], where only linear optical elements are required. In the present experiment, following the scheme as suggested in ref.[23, 24], we report the first experimental demonstration of entanglement concentration. Meanwhile, using local filtering [22] and entanglement swapping [17] we are also able to report the first experimental verification of a quantum repeater.

We first explain the entanglement concentration scheme (shown in Fig. 1a). Suppose that two parties, Alice and Bob, would like to share photon pairs in the maximally entangled state $\left|\Psi^{+}\right\rangle=1 / \sqrt{2}(|H\rangle|V\rangle+|V\rangle|H\rangle)$. Further suppose that, due to the imperfect quantum channel, what they share are two photon pairs $(1,2)$ and $(3,4)$ in the following unknown states:

$$
\begin{aligned}
& |\Psi\rangle_{12}=\alpha\left|H_{1}\right\rangle\left|V_{2}\right\rangle+\beta\left|V_{1}\right\rangle\left|H_{2}\right\rangle, \\
& |\Psi\rangle_{34}=\alpha\left|H_{3}\right\rangle\left|V_{4}\right\rangle+\beta\left|V_{3}\right\rangle\left|H_{4}\right\rangle,
\end{aligned}
$$

where Alice holds photons 1 and 3, and Bob holds photons 2 and 4. Before concentration, the polarization of photon 4 is rotated by $90^{\circ}$ using one half-wave plate $\left(R_{90}\right.$ in Fig. 1a). 
After passing through the half-wave plate, the state of photons 3 and 4 becomes

$$
|\Psi\rangle_{34}=\alpha\left|H_{3}\right\rangle\left|H_{4}\right\rangle+\beta\left|V_{3}\right\rangle\left|V_{4}\right\rangle
$$

Then Bob superposes photons 2 and 4 onto a polarization beam splitter (PBS in Fig. 1a). Since the PBS transmits horizontal and reflects vertical polarization, a coincidence detection between the two outputs $2^{\prime}$ and $4^{\prime}$ implies that either both photons 2 and 4 are horizontally polarized or both vertically polarized. Therefore, by selecting those events where there is one and only one photon in the output mode $4^{\prime}$, Alice and Bob can obtain a conditioned four-photon Greenberger-Horne-Zeilinger (GHZ) state [18]

$$
|\Psi\rangle_{c}=\frac{1}{\sqrt{2}}\left(\left|H_{1}\right\rangle\left|V_{2}^{\prime}\right\rangle\left|V_{3}\right\rangle\left|V_{4}^{\prime}\right\rangle+\left|V_{1}\right\rangle\left|H_{2}^{\prime}\right\rangle\left|H_{3}\right\rangle\left|H_{4}^{\prime}\right\rangle\right),
$$

with a probability of $2|\alpha \beta|^{2}$. By further performing a polarization measurement on each of the photons 3 and $4^{\prime}$ in the $+/$ - basis, where $| \pm\rangle=1 / \sqrt{2}(|H\rangle \pm|V\rangle)$, Alice and Bob can then project the photons 1 and $2^{\prime}$ onto the maximally entangled state [23]

$$
\left|\Psi^{+}\right\rangle_{12^{\prime}}=\frac{1}{\sqrt{2}}\left(\left|H_{1}\right\rangle\left|V_{2^{\prime}}\right\rangle+\left|V_{1}\right\rangle\left|H_{2^{\prime}}\right\rangle\right) .
$$

The scheme above deserves some further comments. Comparing with the original concentration scheme by Bennett et al. 20], the present scheme involves only two entangled photon pairs. Hence, it is reachable using the techniques developed in the recent four-photon experiment [18]. Furthermore, the two pairs are required to have the same coefficients at the same experimental trial, but for different trials the coefficients can be different. In reality, this can well be the case if the sources $S$ emit only two entangled pairs at each time and thus only these two pairs experience the same environment. Finally, but more importantly, the same idea can also be used to implement a simplified version of quantum repeaters.

To see how it works, suppose that there is an intermediate station, Charlie, between Alice and Bob (see Fig. 1b). Further suppose that Alice and Charlie share the pair $(1,2)$ and, Charlie and Bob share the pair $(3,4)$. Here, the pairs $(1,2)$ and $(3,4)$ are in the states as shown in equation (1). After the photon 4 passes through the $\mathrm{R}_{90}$, Charlie superposes the photons 2 and 4 at the PBS. Similarly, by performing a polarization measurement on each of the two outputs $2^{\prime}$ and $4^{\prime}$ in the $+/$ - basis, Alice and Bob can thus obtain the maximally entangled state

$$
\left|\Psi^{+}\right\rangle_{13}=\frac{1}{\sqrt{2}}\left(\left|H_{1}\right\rangle\left|V_{3}\right\rangle+\left|V_{1}\right\rangle\left|H_{3}\right\rangle\right) .
$$


In this protocol, both entanglement concentration and entanglement swapping are accomplished in one step. Of course, if the two entangled pairs have different coefficients, for example, the pair $(1,2)$ in the state $\alpha_{12}\left|H_{1}\right\rangle\left|V_{2}\right\rangle+\beta_{12}\left|V_{1}\right\rangle\left|H_{2}\right\rangle$, and the pair (3,4) in the state $\alpha_{34}\left|H_{3}\right\rangle\left|V_{4}\right\rangle+\beta_{34}\left|V_{3}\right\rangle\left|H_{4}\right\rangle$, then the above protocol is not applicable. In the latter case, if the coefficients are known in advance, we can first apply local filtering to each of the two pairs, then utilize entanglement swapping to generate maximally entangled state $\left|\Psi^{+}\right\rangle_{13}$. Thus, the task of a quantum repeater can still be accomplished. It is worth noting that although the above scheme is only a simplified variant, the scheme itself acquires all the necessary features as a quantum repeater.

A schematic drawing of the experimental setup of entanglement concentration is shown in Fig. 2a. In the experiment, we first generate two photon pairs in the maximally entangled state $\left|\Psi^{+}\right\rangle$by type II parametric down-conversion [26] from an ultraviolet (UV) pulsed laser in a BBO crystal. The UV pulse passing through the crystal creates the first pair in modes 1 and 2. After retroflection, during its second passage through the crystal, the laser pulse creates the second pair in modes 3 and 4 . The UV pulsed laser with a central wavelength of $394 \mathrm{~nm}$ has a pulse duration of $200 \mathrm{fs}$, a repetition rate of $76 \mathrm{MHz}$, and an average power of $450 \mathrm{~mW}$. To prepare the states in equation (1), we send photons 1 and 3 through the same amount of Brewster's windows (BWs) with vertical axis tilted by $56^{\circ}$. Due to the polarization-dependent reflectivity, the transmitted photons are preferentially horizontally polarized. Here, with one piece of BW, the transmission probability for horizontal polarization is $T_{H}=0.98$, and for vertical one $T_{V}=0.73$.

After photon 4 passes through the half wave plate $R_{90}$, photons 2 and 4 are steered to the PBS where the path lengths of the two photons have been adjusted such that they arrive simultaneously. Through spectral filtering ( $\mathrm{F}$ in Fig. 2) with a $\Delta \lambda_{F W H M}=3.6 \mathrm{~nm}$ for all the four photons, the coherence time of the photons was made to exceed the duration of the UV pulse, thus making the two photons 2 and 4 indistinguishable in time [27]. Furthermore, fiber-coupled single-photon detectors have been used to ensure good spatial mode overlap between 2 and 4 . These arrangements consequently lead to two-photon interference. Through the whole experiment, the registered two-fold coincidence rate is about $2.6 \times 10^{4}$ per second before the Brewster's windows and the PBS, which results in an overall four-fold coincidence of 8 per second, almost two orders of magnitude higher compared to the recent four-photon experiment [18]. 
In our experiment, we choose to insert one, two or four pieces of BWs into each of the modes 1 and 3 to prepare three different initial states (denoted by (a), (b), (c)). Furthermore, in order to demonstrate the protocol works for complex coefficients $\alpha$ and $\beta$ (i.e. for both amplitude and phase errors), we also slightly tilt the birefrigent compensators (not shown in the figure) in the modes 1 and 3 such that a $\pi / 2$ relative phase is introduced between the $|H\rangle|V\rangle$ and $|V\rangle|H\rangle$ components. Ideally, these initial states should only contain $|H\rangle|V\rangle$ and $|V\rangle|H\rangle$ terms. However, in reality all the four possible outcomes in the $H / V$ basis have been observed. Correspondingly, the fractions of the four components are shown in Figs. 3a, 3b and 3c. In all three caess, the signal-to-noise ratio is better than 100:1. In addition, we also list in Table 1 the ratios between $|H\rangle|V\rangle$ and $|V\rangle|H\rangle$.

If entanglement concentration works, then conditional on a $|+\rangle|+\rangle$ coincidence detection in the modes 3 and $4^{\prime}$, the remaining two photons 1 and $2^{\prime}$ would be projected onto the maximally entangled state $\left|\Psi^{+}\right\rangle_{12^{\prime}}$. To verify this prediction, we first measure the fractions of the photons 1 and $2^{\prime}$ in the $H / V$ basis after entanglement concentration. The measurement results are shown in Figs. 3d, 3e and 3f, respectively, from which we can see the signal-tonoise ratios are larger than 20:1 for all three cases. Therefore, the contributions of $|H\rangle|H\rangle$ and $|V\rangle|V\rangle$ terms are negligible. At the same time, in Table 1 we also list the ratios between $|H\rangle|V\rangle$ and $|V\rangle|H\rangle$ after concentration. Compared with the initial ratios, one can clearly see the significant improvement in the relative intensity between $|H\rangle|V\rangle$ and $|V\rangle|H\rangle$.

Showing that both $|H\rangle|V\rangle$ and $|V\rangle|H\rangle$ appear with roughly the same probability of $50 \%$ is just a necessary but not sufficient condition to verify the state $\left|\Psi^{+}\right\rangle_{12^{\prime}}$, since the above observation is, in principle, both compliant with $|\Psi\rangle_{12^{\prime}}$ and with a statistical mixture of $|H\rangle|V\rangle$ and $|V\rangle|H\rangle$. Thus, as a further test we have to demonstrate that the two terms $|H\rangle|V\rangle$ and $|V\rangle|H\rangle$ are indeed in a coherent superposition. To do so, we further perform a polarization analysis in the $+/$ - basis. Transforming $\left|\Psi^{+}\right\rangle_{12^{\prime}}$ to the $+/-$ basis yields an expression containing only $|+\rangle|+\rangle$ and $|-\rangle|-\rangle$, but no other terms like $|+\rangle|-\rangle$ and $|-\rangle|+\rangle$. As a test for coherence we can now check the presence or absence of various components. In Fig. 4 we compare the $|+\rangle|+\rangle$ and $|-\rangle|+\rangle$ count rates as a function of the pump delay mirror position conditional on detecting a $|+\rangle|+\rangle$ coincidence in the modes 3 and $4^{\prime}$. At zero delay - photons 2 and 4 arrive at the PBS simultaneously - the latter component is suppressed with a visibility of $0.83 \pm 0.04,0.80 \pm 0.05$ and $0.80 \pm 0.04$, respectively.

The high visibility observed in the $+/-$ basis implies a violation of a suitable Bell's 
inequality for the photons 1 and $2^{\prime}$. According to the inequality of Clauser-Horne-ShimonyHolt (CHSH) [28], there is a constraint on the value of $S$, a combination of four polarization correlation probabilities - two possible analysis settings for each photon. If $|S| \leq 2$, no quantum entanglement is necessary to explain the correlations, that is, some local realistic model can reproduce them. For the maximally entangled state $\left|\Psi^{+}\right\rangle_{12^{\prime}}$, the maximum value of $S$ is $2 \sqrt{2}$. Therefore, the value of $S$ is a figure of merit for the quality of the entanglement between the photons 1 and $2^{\prime}$. In the experiment, we also measured the $S$ value for all three cases. The integration time is about $10^{3}$ seconds for each possible outcomes in the first two cases, and $2 \times 10^{3}$ seconds in the third case. The CHSH inequality was violated by more than 5 standard deviations in all cases; see Table 1 . Since the entanglement visibility $V$ satisfies the relation $V=S / 2 \sqrt{2}$, we can calculate the visibility $V$ from the observed value $S$ and further estimate the fidelity for the photons 1 and $2^{\prime}$ to be in the state $\left|\Psi^{+}\right\rangle_{12^{\prime}}$. As shown in Table 1, the fidelity obtained is larger than 0.93 in all cases.

In order to show our experiment also provides an realization of a quantum repeater, we reassemble the setup as shown in Fig. 2b. We first consider the case where the two photon pairs $(1,2)$ and $(3,4)$ are in the same initial state. In this case, as discussed in equation (5), conditional on detecting a $|+\rangle|+\rangle$ coincidence in $2^{\prime}$ and $4^{\prime}$, the photons 1 and 3 shared by Alice and Bob will be left in the state $\left|\Psi^{+}\right\rangle_{13}$. In the first repeater experiment, we choose the initial state (b) to verify this prediction. This is done by measuring the fractions of the photons 1 and 3 in both $H / V$ and $+/-$ bases. While $\mathrm{HV}$ and $\mathrm{VH}$ components are observed with roughly the same probability of $50 \%$, both $\mathrm{HH}$ and VV terms are negligible. In addition, an interference visibility of $0.81 \pm 0.04$ is observed in the $+/-$ basis. The $S$ value for the photons 1 and 3 is measured to be $2.44 \pm 0.08$, from which the fidelity for the photons 1 and 3 to be in the state $\left|\Psi^{+}\right\rangle_{13}$ is about $0.93 \pm 0.04$.

We now consider the case that the two photon pairs $(1,2)$ and $(3,4)$ are in different initial states. In the second repeater experiment, we first prepare the pair (1,2) in the state (a) and the pair $(3,4)$ in the state $(\mathrm{b})$. We then perform local filtering to convert the pairs $(1,2)$ and $(3,4)$ into the same maximally entangled state $\left|\Psi^{+}\right\rangle$. Finally, we utilize entanglement swapping to generate the desired state $\left|\Psi^{+}\right\rangle_{13}$ between Alice and Bob. Again, this is achieved by detecting a $|+\rangle|+\rangle$ coincidence in $2^{\prime}$ and $4^{\prime}$. To verify the success of our quantum repeater protocol, we also measured the $S$ value for the photons 1 and 3, which is $2.52 \pm 0.10$. This implies that the fidelity for the photons 1 and 3 to be in the state $\left|\Psi^{+}\right\rangle_{13}$ is about $0.95 \pm 0.05$. 
It should be mentioned that in the above repeater experiments both entanglement purification and entanglement swapping play a crucial role in generating maximally entangled states between Alice and Bob. Without applying entanglement concentration or local filtering, the operation of entanglement swapping alone will only generate a non-maximally entangled state, say, $\alpha^{2}|H\rangle|V\rangle+\beta^{2}|H\rangle|V\rangle$ in the first repeater experiment. Such a state acquires an entanglement fidelity even worse than the original pairs. The necessity of both entanglement concentration (or local filtering) and entanglement swapping implies that both experiments present a faithful demonstration of a quantum repeater. Furthermore, after taking into account the imperfection of the $\left|\Psi^{+}\right\rangle$state preparation in the scond experiment, we can estimate the PBS accuracy to interfere two independent photons from the experimental visibility for entanglement swapping, which is about $98 \%$. This clearly surpasses the strict precision requirement of local operations for the multi-stage realization of quantum repeaters 16]. Therefore, the techniques developed for our entanglement concentration and quantum repeater experiment provide a practical toolbox for future realization of long-distance quantum communication.

[1] C. H. Bennett \& S. J. Wiesner, Phys. Rev. Lett. 69, 2881 (1992).

[2] K. Mattle, H. Weinfurter, P. G. Kwiat \& A. Zeilinger, Phys. Rev. Lett.76, 4656 (1996).

[3] C. H. Bennett et al., Phys. Rev. Lett. 70, 1895 (1993).

[4] D. Bouwmeester et al., Nature 390, 575 (1997).

[5] D. Boschi, S. Branca, F. De Martini, L. Hardy \& S. Popescu, Phys. Rev. Lett. 80, 1121 (1998).

[6] J.-W. Pan, S. Gasparoni, M. Aspelmeyer, T. Thomas \& A. Zeilinger. Nature, in press.

[7] C. H. Bennett \& G. Brassard, in Proc. IEEE Int. Conf. on Computers, Systems and Signal Processing, 175 (IEEE, New York, 1984).

[8] A. K. Ekert, Phys. Rev. Lett. 67, 661 (1991).

[9] T. Jennewein, C. Simon, G. Weihs, H. Weinfurter \& A. Zeilinger, Phys. Rev. Lett. 84, 4729 (2000).

[10] D. S. Naik, C. G. Peterson, A. G. White, A. J. Berglund \& P. G. Kwiat, Phys. Rev. Lett. 84, $4733(2000)$.

[11] W. Tittel, T. Brendel, H. Zbinden \& N. Gisin, Phys. Rev. Lett. 84, 4737 (2000). 
[12] N. Gisin, G. Ribordy, W. Tittel \& H. Zbinden, Rev. Mod. Phys. 74, 145 (2002).

[13] M. Zukowski, A. Zeilinger, M. A. Horne \& A. Ekert, Phys. Rev. Lett. 71, 4287 (1993).

[14] C. H. Bennett et al, Phys. Rev. Lett. 76, 722 (1996).

[15] D. Deutsch et al, Phys. Rev. Lett. 77, 2818 (1996).

[16] H.-J. Briegel, W. Duer, J.-I. Cirac \& P. Zoller, Phys. Rev. Lett. 81, 5932 (1998).

[17] J.-W. Pan, D. Bouwmeester, H. Weinfurter, \& A. Zeilinger, Phys. Rev. Lett. 80, 3891 (1998).

[18] J.-W. Pan, M. Daniell, S. Gasparoni, G. Weihs \& A. Zeilinger, Phys. Rev. Lett. 86, 4435(2001).

[19] T. Jennewein, G. Weihs, J.-W. Pan \& A. Zeilinger, Phys. Rev. Lett. 88, 017093 (2002).

[20] C. H. Bennett, H. J. Bernstein, S. Popescu \& B. Schumacher, Phys. Rev. A53, 2046 (1996).

[21] M. Horodecki, P. Horodecki \& R. Horodecki, Phys. Rev. Lett. 78, 547 (1997).

[22] P. G. Kwiat, S. Barraza-Lopez, A. Stefanov \& N. Gisin, Nature 409, 1014 (2001).

[23] Z. Zhao, J.-W. Pan \& M. S. Zhan, Phys. Rev. A 64, 014301 (2001).

[24] T. Yamamoto, M. Koashi \& N. Imoto, Phys. Rev. A 64, 012304 (2001).

[25] J.-W. Pan, C. Simon, C. Brukner \& A. Zeilinger, Nature 410, 1067 (2001).

[26] P. G. Kwiat et al, Phys. Rev. Lett. 75, 4337 (1995).

[27] M. Zukowski, A. Zelinger \& H. Weinfurter, Ann. NY Acad. Sci. 755, 91 (1995).

[28] J. Clauser, M. Horne, S. Shimony \& R. Holt, Phys. Rev. Lett. 23, 880 (1969).

\section{Acknowledgements}

This work was supported by the National Natural Science Foundation of China, the Chinese Academy of Sciences and the National Fundamental Research Program (under Grant No. 2001CB309303).

* To whom correspondence should be addressed. E-mail:pan@ap.univie.ac.at 
Table 1 Summary of entanglement concentration data for different BWs

\begin{tabular}{ccccc}
\hline Number of BWs Pre-concentration After concentration & $S$ values & Fidelities \\
\hline 1 & $1.41: 1$ & $1.08: 1$ & $2.58 \pm 0.07$ & $0.96 \pm 0.04$ \\
2 & $1.72: 1$ & $1.09: 1$ & $2.43 \pm 0.08$ & $0.93 \pm 0.04$ \\
4 & $3.15: 1$ & $1.10: 1$ & $2.42 \pm 0.08$ & $0.93 \pm 0.04$ \\
\hline
\end{tabular}

\section{Figure Captions:}

Figure captions:

Figure 1: Schematic drawings showing the principles of entanglement concentration (a) and quantum repeater $(\mathbf{b})$. a, The sources $\mathrm{S}$ emit two entangled photon pairs into the modes $(1,2)$ and $(3,4)$. The two pairs are originally in the same unknown non-maximally entangled state. One member of each pair is then sent to Alice, and the other one to Bob. After photon 4 passes through the half wave plate $R_{90}$, Bob superposes the photons at the polarizing beam splitter PBS. By performing a polarization measurement on each of the photons in mode 3 and $4^{\prime}$ in the $+/-$ basis, Alice and Bob can project the photons 1 and $2^{\prime}$ onto a maximally entangled state. b, Alice and Charlie share the pair $(1,2)$ and, Bob and Charlie share the pair $(3,4)$. Interfering the photons 2 and 4 at the PBS and performing a subsequent polarization measurement on each of the modes $2^{\prime}$ and $4^{\prime}$ in the $+/-$ basis, Charlie can thus generate a maximally entangled state between Alice and Bob (see text).

Figure 2: The experimental setups for entanglement concentration (a) and quantum repeater (b). a, A UV pulse passes through a BBO twice to generate two entangled photon pairs. By inserting one, two or four pieces of Brewster's windows (BWs) into each of the modes 1 and 3, we can prepare three different nonmaximally entangled states. After the photons 2 and 4 pass through the PBS, conditional on a two-fold coincidence detection behind the two $45^{\circ}$ polarizers Pol in the modes 3 and $4^{\prime}$, the photons 1 and $2^{\prime}$ will be in a maximally entangled state. $\mathbf{b}$, If the pairs $(1,2)$ and $(3,4)$ are in the same nonmaximally entangled state, then detecting a two-fold coincidence behind the two $45^{\circ}$ polarizers Pol in the modes $2^{\prime}$ and $4^{\prime}$ is sufficient to project the photons 1 and 3 onto the desired maximally entangled state. Otherwise, additional half wave plates and BWs are needed in the modes 1 and 3 to generate the desired entangled state between Alice and Bob.

Figure 3: Experimental results demonstrating the entanglement concentration. $\mathbf{a}$, $\mathbf{b}$, and c show the fractions of the nonmaximally entangled state in the $H / V$ basis, when inserting 
one, two and four BWs into the modes 1 and 3. $\mathbf{d}$, e, and $\mathbf{f}$ show the corresponding fractions of the photons 1 and $2^{\prime}$ in the $H / V$ basis after entanglement concentration. The data clearly confirm that our concentration protocol significantly improved the relative intensity between $|H\rangle|V\rangle$ and $|H\rangle|V\rangle$ components.

Figure 4: Experimental results confirming the entanglement between the photons 1 and $2^{\prime}$. The two-fold coincidence count rate $|+\rangle|+\rangle$ and $|-\rangle|+\rangle$ is shown as a function of the pump delay mirror position under the condition that a $|+\rangle|+\rangle$ coincidence is registered in the modes 3 and $4^{\prime}$. 
(a)

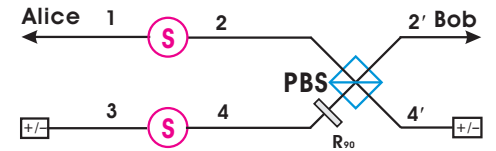

Alice (b)

FIG. 1: 
(a)
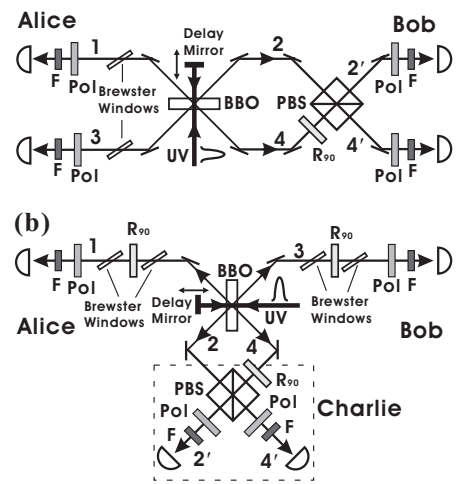

FIG. 2: 

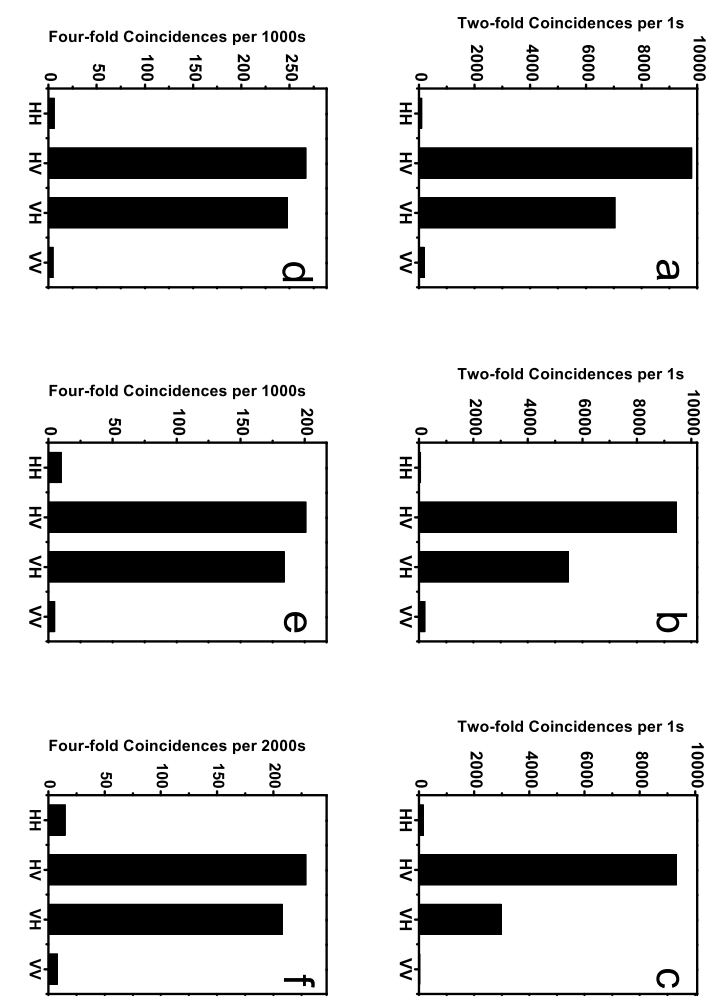

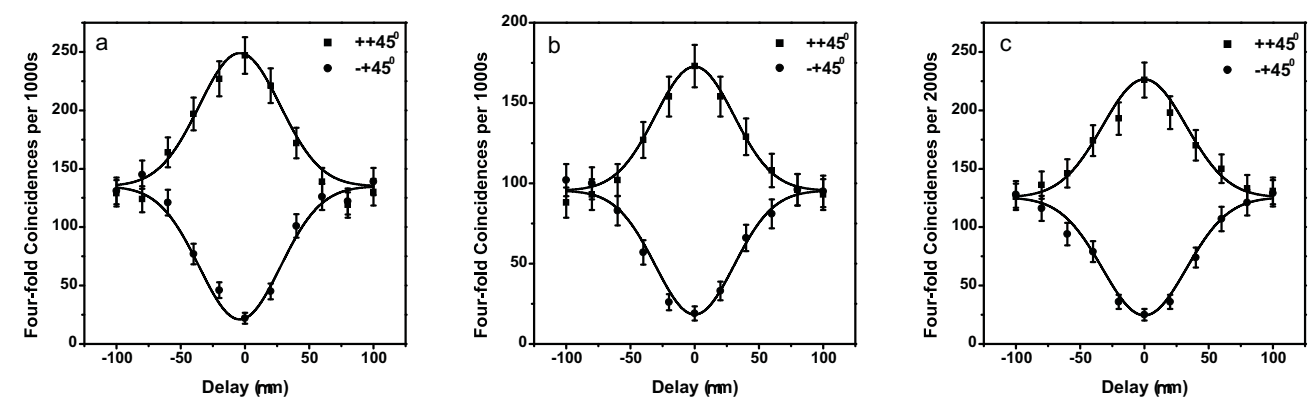

FIG. 4: 